\title{
ESTUDO FITOSSOCIOLÓGICO NAS REGIÕES DE CARAJÁS E MARABÁ - PARA, BRASIL
}

\author{
Ralfh João RIBEIRO' , Niro HIGUCHI', Joaquim dos SANTOS' e Celso Paulo \\ de AZEVEDO².
}

RESUMO - O estudo fitossociológico levou em consideração duas áreas localizadas em Carajás e Marabá, na região sul do Pará. A amostragem abordou dois níveis; 1) Individuos com DAP $\geq 20 \mathrm{~cm}$ em parcelas de $20 \mathrm{~m} \times 200 \mathrm{~m}$ e, II) Indivíduos com $5 \mathrm{~cm} \leq$ DAP $<20 \mathrm{~cm}$ em duas sub-parcelas de $10 \mathrm{~m}$ $x 10 \mathrm{~m}$ no início e final das parcelas. Todos os indivíduos do segundo nível foram considerados como regeneração natural do povoamento adulto. Em Carajás utilizou-se 35 (trinta e cinco) unidades amostrais (parcelas) e em Marabá 22 (vinte e duas). Do ponto de vista de composição florística, as áreas estudadas apresentaram-se heterogêneas, com índices de Shanon-Weaver estimados em 3,66 e 3,71, respectivamente, para Carajás e Marabá. A equação hipsométrica comum que melhor ajustou os dados observados foi: $h=\left[\frac{d}{2,38+0,1387 h}\right]^{2}$. As abundâncias médias foram 131,92 e 127,85 indivíduos/ ha, respectivamente, para Carajás e Marabá. As estimativas de área basal foram 15,41 e 17,35 m²/ha nas regiões de Carajás e Marabá, respectivamente. A função de densidade 1 (Meyer) que melhor ajustou os dados de freqüência por classe de diâmetro $\left(\mathrm{F}_{i}\right)$ em função do centro de classe (DAP), para Carajás, foi $\mathrm{F}_{i}$ $=\mathrm{e}^{(9.56330614-0.07139847 \mathrm{~d})}$ e $\mathrm{F}_{\mathrm{i}}=\mathrm{e}^{(8.96125691-0.06937877 \mathrm{~d})}$ para Marabá. Também estimou-se que cerca de $36 \%$ das espécies existentes nas regiōes estudadas possuem valor econômico no mercado madeireiro.

Palavras-chave: Análise fitossociológica, inventário florestal, manejo florestal.

\section{Plant Structural Analysis on Carajás and Marabá Regions - PARA State, Brazil}

ABSTRACT - The structural analysis was carried out in Carajás and Marabá regions, South of State of Pará. The sampling plots of the forest inventory were divided in two levels: I) main sample plot with $20 \mathrm{~m}$ $\times 200 \mathrm{~m}$ for all trees with $\mathrm{dbh} \geq 20 \mathrm{~cm}$ and, II) sub-sample plot with $10 \mathrm{~m} \times 10 \mathrm{~m}$ for all trees with $5 \mathrm{~cm} \leq \mathrm{DAP}<20 \mathrm{~cm}$ at the beginning and at the end of the main sample. All trees of the level II were considered as natural regeneration of the studied forest. In Carajás were set up 35 (thirty five) sample plots and 22 (twenty two) in Marabá region. The Shanon-Weaver diversity indexes were 3.66 for Marabá and 3.71 for Carajás region. The observed data were better fitted by the following hypsometric function (relationship between height and $\mathrm{dbh}$ ) : $h=\left[\frac{d}{2.38+0.1387 h}\right]^{2}$. The dominances averaged 131.92 and 127.85 trees/ha for Carajás and Marabá, respectively. The basal areas averaged 15.41 and $17.35 \mathrm{~m} 2 /$ ha for Carajás and Marabá. The density functions of Meyer which best fitted the observed data were $F_{i}=$ $\mathrm{e}^{(9.56330614-0.07139847 \mathrm{~d})}$ for Carajás and $\mathrm{F}_{\mathrm{i}}=\mathrm{e}^{(8.96125691-0.06937877 \mathrm{~d})}$ for Marabá. For both regions about $36 \%$ of the inventoried species have some economical market value.

Key-words: Phytosociological study, forest inventory, forest management

\section{INTRODUÇÃO}

A floresta amazônica é composta de um conjunto de ecossistemas complexos, heterogêneos e frágeis, sobre solos pobres em nutrientes e ácidos. Por esta razão, as atividades de manejo nessa região têm que ser respaldadas no conhecimento dos seus ecossistemas.

A análise da estrutura florestal ainda é a técnica mais utilizada para se fazer deduções sobre a origem, características ecológicas e sinecológicas, dinâmica e tendência sobre seu futuro desenvolvimento. A posse destas informações permite a definição acerca da técnica de

1 Instituto Nacional de Pesquisas da Amazônia - Cx. Postal, 478 - Manaus - Am.

2 Empresa Brasileira de Pesquisa Agropecuária - Km 26 - AM/010-Manaus - Am. 
manejo a ser adotado, principalmente no que concerne às intervenções silviculturais.

A estrutura caracteriza a distribuição dos indivíduos que compõem a associação. Verticalmente: pela estratificação, isto é, a divisão da massa vegetal em estratos determinado por limite de altura. Horizontalmente: pela cobertura, ou seja, a percentagem do solo coberto pela projeção perpendicular de cada estrato, e consistência ou textura sendo definida por caracteristicas como penetrabilidade, flexibilidade, resistência etc, segundo Dansereau (1961), citado por Montoya Maquin (1966).

A partir da estimativa de valores relativos de densidade, dominância e freqüência, foi introduzido por Curtis (1951), o "Índice de Valor de Importância (IVI)" que passou a ser expresso como a soma dessas três estimativas.

A utilização apenas do IVI, no entanto, limitando-se à análise de sua estrutura horizontal, não permite uma real definição da importância ecológica da espécie. Finol (1971) propôs a inclusão da estrutura vertical com a finalidade de corrigir esta discrepância, passando a utilizar a estimativa da posição sociológica através da subdivisão do povoamento em três estratos de altura (inferior, médio e superior), a classe de tamanho e a regeneração natural relativa, que é dada pela média aritmética dos valores relativos de abundância, freqüência e classe de tamanho da regeneração natural.

Quanto a intensidade amostral, o minimo suficiente para descrever a estrutura de um povoamento com árvores de DAP $>10 \mathrm{~cm}$, para Finol (1971), foi de 1 ha através da utilização da curva espécie-área. Este resultado foi confirmado por Porto et al. (1976) que efetuou estudos de aspectos fitossociológicos em floresta de baixio, baseados no gráfico de área mínima.

Na Amazônia, Jardim \& Hosokawa (1986/87) concluíram que, em áreas superiores a $190 \mathrm{ha}$, para individuos com $\mathrm{DAP}>20 \mathrm{~cm}$ são necessários um mínimo de 7 hectares para abordagem quantitativa e um minimo de 2 hectares para a qualitativa em unidades amostrais, preferencialmente, de $20 \mathrm{~m} \times 500 \mathrm{~m}$; e para individuos com DAP entre $5 \mathrm{~cm}$ e $20 \mathrm{~cm}$, em áreas superiores a 190 ha, a utilização de no minimo 18 unidades amostrais para avaliação quantitativa e um mínimo de 6 unidades amostrais para abordagem qualitativa em unidades amostrais de preferência de $10 \mathrm{~m} \times 100 \mathrm{~m}$.

O presente trabalho tem como objetivo analisar a estrutura de uma floresta típica da região Sul do Pará, como pressuposto na tomada de decisões em planos de manejo florestal em regime de rendimento sustentado.

\section{MATERIAIS E MÉTODOS}

\section{Caracterização da Área de Estudo}

As duas áreas de estudo estão localizadas nos municípios de Carajás e Marabá. A micro-região de Carajás localiza-se no sul do Estado do Pará e Norte do Estado de Goiás, na inferência das bacias dos rios Araguaia-Tocantins e Xingu, com uma superficie em torno de $150.000 \mathrm{~km}^{2}$.

A serra dos Carajás está situada a $130 \mathrm{~km}$ a oeste de Marabá sendo banhado pelas bacias dos rios Itacaiuanas e Parauapebas. A micro- 
região de Marabá situa-se entre os rios Itacaiunas e Tocantins. As elevações variam de 600 a $800 \mathrm{~m}$ (Cunha et al., 1985). As duas micro-regiões localizam-se entre os paralelos $5^{\circ} 00^{\prime}$ e $6^{\circ} 00^{\prime} \mathrm{S}$ e os meridianos $49^{\circ} 00^{\prime}$ e $50^{\circ} 00^{\prime} \mathrm{WG}$.

O clima das duas micro-regiões é tipicamente tropical, quente e úmido, clásicado por Köppen como sendo do tipo "AWi" com uma estação seca cuja precipitação média do mês mais seco é inferior a $60 \mathrm{~mm}$ e por apresentar variação de temperatura média anual inferior a $5^{\circ} \mathrm{C}$ (Pereira, 1991). Segundo o mesmo autor as variações termais ficam em torno de $24,3^{\circ} \mathrm{C}$ a $28,3^{\circ} \mathrm{C}$. A amplitude térmica entre o mês mais frio e o mês mais quente é inferior a $3^{\circ} \mathrm{C}$.

As médias de precipitação na microregião de Carajás, no periodo de 1969 1984, ficaram em torno da máxima de $3.139 \mathrm{~mm}$, mínima igual a $1.394 \mathrm{~mm}$ e média de $2.116 \mathrm{~mm}$ (Relatório CVRD, 1980). Em Marabá no periodo de $1977-$ 1986 as precipitações médias ficaram em torno da máxima de $2.256 \mathrm{~mm}$, mínima igual a $1.675 \mathrm{~mm}$ e média de $2.024 \mathrm{~mm}$ (Pereira, 1991).

Segundo o atlas do Conselho Interministerial do Programa Grande Carajás (1981), a maioria dos solos enquadra-se no tipo Podzólico Vermelho Amarelo, com maior freqüência a margem esquerda do Rio Tocantins a partir do paralelo $3^{\circ} \mathrm{S}$, podendo ocorrer a presença de latossolos vermelho amarelo (Pereira, 1991).

A vegetaçâo da região de Tocantins foi descrita por Hueck (1972) como floresta pluvial e mencionou a presença de "campos abertos". Pereira (1991) classificou-a como floresta densa, ombrófila tropical, floresta tropical chuvosa representada pela sub-região da superficie dissecada do Araguaia, onde árvores densas e altas são intercaladas por outras que emergem do dossel normal da floresta ( $\pm 40 \mathrm{~m}$ de altura).

Fisionomicamente, a vegetação pode ser descrita como sendo uma floresta mista de cipós com pequenas manchas, mais densas sem cipós, assemelhando-se à floresta densa submontana - baixa e uniforme (Salomão et al., 1989). O Relatório do Museu Goeldi (1988) classificou em dois grandes grupos: floresta tropical pluvial e savana metalófila ou campo rupestre ou simplesmente vegetação de canga. Segundo Ab'Saber (1986), a área florestal ocupa um espaço superior a $95 \%$ do total, o restante em torno de 2 a 3\% são formados por clareiras naturais de vegetação rupestre.

\section{Amostragem e Coleta dos Dados}

O sistema de amostragem adotado na coleta de dados foi em dois estágios. As unidades primárias foram tomadas aleatoriamente e as secundárias de forma sistemática (Husch et al., 1972 e Péllico Neto, 1982). Adotou-se a abordagem da população em dois níveis: o primeiro nivel para os individuos com $\mathrm{DAP} \geq 20 \mathrm{~cm}$ e o segundo nivel para árvores entre $5 \mathrm{~cm}$ e $20 \mathrm{~cm}$ de DAP.

A forma e o tamanho das unidades amostrais foram estabelecidas de modo sistemático no campo conforme Higuchi et al. (1982), comprovadas por Scolforo et al. (1993) da seguinte maneira: forma retangular de $20 \mathrm{~m} \times 200 \mathrm{~m},(0,4 \mathrm{ha})$ cada, com duas sub-amostras de $10 \mathrm{~m} \times 10 \mathrm{~m}$ ( $0,01 \mathrm{ha}$ ) em lados inversos no inicio e fim da unidade de amostra (Fig.1). Fo- 
ram medidas 22 (vinte e duas) unidades amostrais em Marabá e 35 (trinta e cinco) em Carajás (Tab. 1).

Em cada amostra, foram coletados os seguintes dados: nome vulgar e DAP de todos os indivíduos nos dois niveis de abordagem e altura de 87 árvores na região de Carajás e 97 em Marabá para a geração de relação hipsométrica e posterior estimativa das alturas das demais árvores amostradas. Na estimativa das relações hipsométricas foram testados modelos aritméticos e logaritmicos, sendo as funções utilizadas identificadas a seguir:

1) Modelo parabólico

II) Modelo de Curtis

III) Modelo de Petterson

IV) Modelo de Naslund

V) Modelo de Heenriksen

V1) Modelo de Prodan

VII) Modelo de Stoffels

\section{Parâmetros Florísticos e Fitossociológicos}

A composição floristica foi analisada através da distribuição de indivíduos, espécies, gêneros e famílias botânicas que ocorreram nas áreas. Também foi utilizado o Índice de diversidade floristica de ShannonWeaver (Poole, 1974). Na estimativa dos padrões de dispersão utilizou-se o indice de McGuinnes, como descrito por Carvalho (1982), Vieira (1987), Flores (1993) e Calegário (1993). Para comparar a composição especifica de duas comunidades foi utilizado o Índice de Sorensen (Horn, 1966).

A análise fitossociológica envolveu as estimativas dos parâmetros da estrutura horizontal e vertical, de maneira a conhecer a importância de cada espécie na referida comunidade.

As estimativas dos parâmetros da estrutura horizontal incluiram a freqüência, que mede a distribuição de cada espécie, em termos percentuais sobre a área; densidade ou abur Hância, que é o número de indivíduos de caúa espécie na composição florística do povoamento; e a que pode ser definida como a medida da projeção do corpo da planta no solo. Estes parâmetros, quando reunidos em uma única expressão, proporcionam o indice de valor de importância e o indice de valor de cobertura.

Na determinação dos parâmetros da estrutura vertical, foram feitas estimativas da Posição Sociológica e Regeneração Natural, conforme recomendado por Finol (1971).

A posição sociológica, para Lamprecht (1964), diz respeito ao lugar que esta ocupa na estrutura vertical de uma floresta. Esta estrutura fornece a distribuição das espécies em diferentes estratos, possibilitando analisar a composição florística verticalmente. Para a obtenção das estimativas de Posição sociológica (absoluta e relativa) dą iésima espécie, apenas para o nível I da amostragem, dividiu-se a amostra em três estratos (Tab. 2), segundo procedimento recomendado por Jardim \& Hosokawa (1986/87).

Para obtenção da categoria de tamanho absoluta da regeneração natural (nivel II) utilizou-se critério sugerido por Barnard (1950), adotado por Finol (1971) e aplicado por Longhi (1980), 

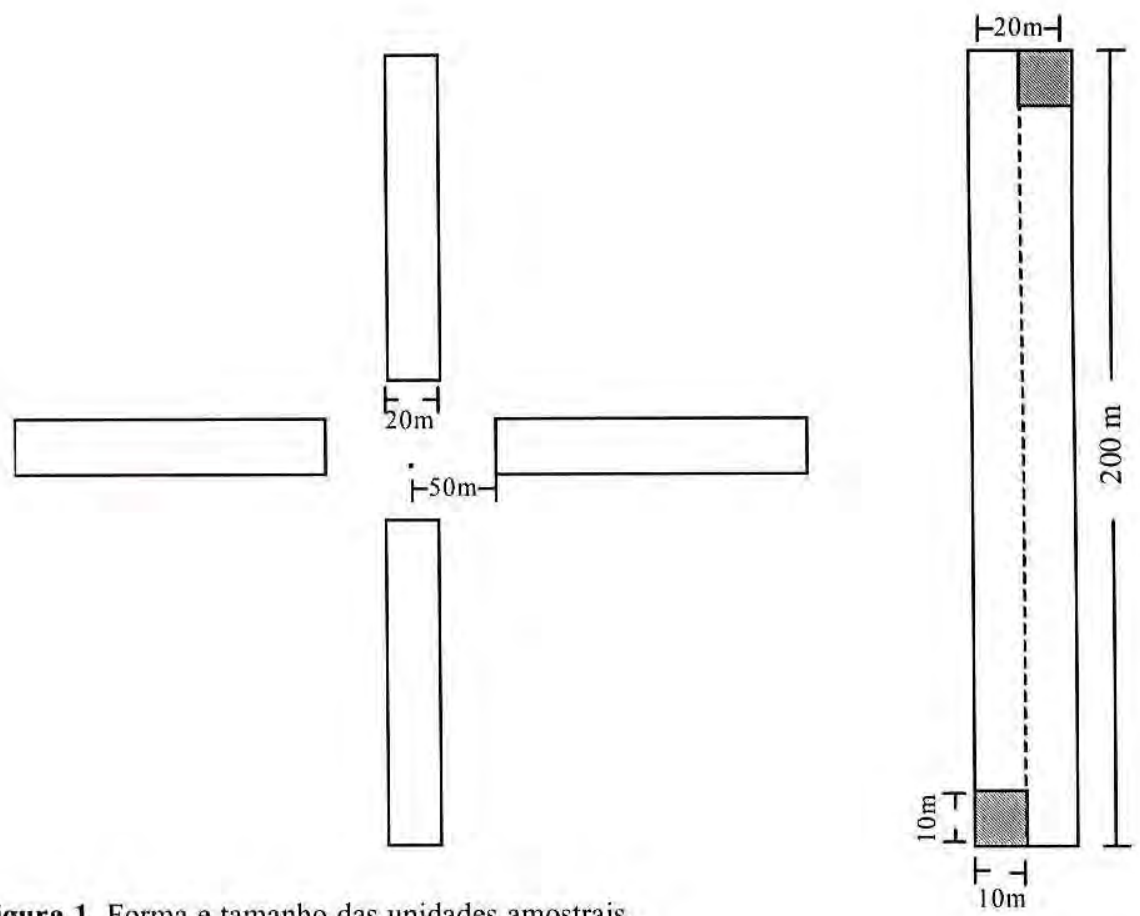

Figura 1. Forma e tamanho das unidades amostrais.

Tabela 1. Locais amostrados e número de unidades amostrais (UA).

\section{LOCALIDADE}

№ AMOSTRAS

Fazenda Pioneira, COSIPAR, $40 \mathrm{Km}$ SO Marabá 8

Fazenda São José, COSIPAR, 45 Km SO Marabá 6

Reserva Florestal, CVRD, 45 Km SO Marabá 8

Área Exploração de Ferro, Carajás, 100 Km SE Marabá

Área Proteção Ambiental, Carajás, 100 Km SE Marabá 4

Reserva Biológica Tapirapé, Carajás, 100 Km SE Marabá 7

Floresta Nacional Tapirapé-Aquiri, 100 Km SE Marabá 20

Fonte: Higuchi et. al. 1992.

Tabela 2. Estratificação do povoamento.

\begin{tabular}{lc}
\hline \multicolumn{1}{c}{ ESTRATO } & INTERVALO DE DIÂMETRO (CM) \\
\hline Estrato inferior (I) & $20 \leq \mathrm{DAP}<50$ \\
Estrato médio (II) & $50 \leq \mathrm{DAP}<80$ \\
Estrato superior (III) & $\mathrm{DAP} \geq 80$
\end{tabular}


Carvalho (1982), Higuchi et al. (1985) e Jardim \& Hosokawa (1986/87), com modificações no número de classes para três em virtude da necessidade de adequação aos dados coletados. Tendo sido portanto definidas as seguintes classes de tamanho (Tab. 3):

Tabela 3. Classe de tamanho da regeneração natural.

CLASSE DE TAMANHO AMPLITUDE DA CLASSE

\begin{tabular}{cl}
\hline I & $5 \mathrm{~cm} \leq \mathrm{DAP}<10 \mathrm{~cm}$ \\
II & $10 \mathrm{~cm} \leq \mathrm{DAP}<15 \mathrm{~cm}$ \\
III & $15 \mathrm{~cm} \leq \mathrm{DAP}<20 \mathrm{~cm}$ \\
\hline
\end{tabular}

O parâmetro regeneração natural relativa foi estimado através da média aritmética de três parâmetros, freqüência relativa da espécie $i$ no nivel II; abundância relativa da espécie $\boldsymbol{i}$ no nivel II e classe de tamanho relativa da espécie $i$, conforme recomendação de Finol (1971).

A estimativa do "Índice de Valor de Importância Ampliado (IVIA)" por espécie, engloba os valores dos parâmetros fitossociológicos da estrutura horizontal e vertical, caracterizando melhor a importância ecológica da espécie na comunidade.

\section{RESULTADOS E DISCUSSÃO}

\section{Relação Hipsométrica}

Das funções avaliadas e considerando-se as caracteristicas de cada modelo, foi escolhida para as duas regiões a função de Naslund, Modelo IV (Tab. 4) que, segundo as estatisticas analisadas e a distribuição do resíduo, em percentagem, se mostrou a mais adequada aos dados.

Após este resultado, o teste de identidade de modelos foi construido conforme apresentado por Graybill (1976). Interpretando o significado obtido, concluiu-se pelo emprego da equação comum para estimar as alturas das duas micro-regiões, dada pela função de Naslund, modelo IV, dada pela expressão: $h=\left[\frac{d}{2,3800+0,1387 d}\right]^{2}$

\section{Composição Floristica}

$\mathrm{Na}$ Tabela 5 são apresentadas as espécies florestais que ocorreram nas micro-regiões estudadas em ordem alfabética de nomes vulgares, num total de 130 espécies, das quais, 122 espécies ocorreram nas duas microregiões e apenas oito são exclusivas da micro-região de Carajás.

Nas duas micro-regiões estudadas foram amostrados 4.104 individuos sendo 1.555 individuos na micro-região de Marabá e 2.549 indivíduos na microregião de Carajás pertencentes a 41 familias e 130 espécies. No segundo nivel de abordagem, isto é, na amostragem de 5 DAP 20 foram encontrados $702(27,54 \%)$ individuos na micro-região de Carajás, e 430 (27,65\%) em Marabá. Observou-se também que $55(42 \%)$ das espécies da micro-região de Carajás e 62 (50\%) na micro-região de Marabá sâo exclusivas do primeiro nivel de abordagem, isto é, não apresentam estoque de regeneração natural.

Dentre as famílias com maior número de indivíduos na micro-região de Carajás citamos Fabaceae (20,04\%), Mimosaceae $(13,05 \%)$, Arecaceae (7,09\%) e Lecythidaceae $(3,57 \%)$; em Marabá foram: Fabaceae $(23,38 \%)$, Mimosaceae $(8,89 \%)$, Meliaceae $(8,44 \%)$ e Lecythidaceae $(3,74 \%)$. 
Tabela 4. Características dos modelos de hipsometria analisados.

\begin{tabular}{|c|c|c|c|c|c|c|c|}
\hline Região & Modelo & $F$ & $\mathrm{R}^{2}$ & $\mathrm{~s}_{\mathrm{yx}} \%$ & $\beta_{0}$ & $\beta_{1}$ & $\beta_{2}$ \\
\hline \multirow[t]{7}{*}{ Carajás } & 1 & $122,291^{\star *}$ & 0,7444 & 23,41 & 7,2484 & $0,4694^{\star \star}$ & $-0,0013^{* *}$ \\
\hline & II & $168,106^{\star *}$ & 0,6642 & 89,45 & 3,6636 & $-16,6900$ & - \\
\hline & III & $173,751^{\star *}$ & 0,6715 & 9,24 & 0,2906 & 2,0628 & - \\
\hline & IV & $1809,324^{* *}$ & 0,9551 & 12,66 & 2,5704 & 0,1350 & - \\
\hline & V & $243,914^{\star \star}$ & 0,7416 & 23,40 & $-23,6970$ & 13,3981 & - \\
\hline & VI & $1373,595^{\star \star}$ & 0,9703 & 25,51 & $-1,3954$ & $1,1407^{* *}$ & $0,0150^{* *}$ \\
\hline & VII & $220,163^{\star \star}$ & 0,7215 & 78,94 & 1,0197 & 0,5761 & - \\
\hline \multirow[t]{7}{*}{ Marabá } & 1 & $126,166^{\star \star}$ & 0,7286 & 19,24 & 10,0190 & $0,3905^{\star \star}$ & $-0,0011^{\star \star}$ \\
\hline & II & $143,793^{\star *}$ & 0,6022 & 68,19 & 3,5452 & $-13,0612$ & - \\
\hline & III & $145,397^{\star *}$ & 0,6048 & 7,51 & 0,3040 & 1,5917 & - \\
\hline & IV & $2591,555^{\star \star}$ & 0,9646 & 10,86 & 2,1712 & 0,1436 & - \\
\hline & V & $227,450^{* *}$ & 0,7054 & 19,94 & $-15,3976$ & 11,0003 & - \\
\hline & VI & $2246,531^{\star *}$ & 0,9795 & 20,68 & $-6,4782$ & $1,2599^{\star \star}$ & $0,0153^{\star \star}$ \\
\hline & VII & $193,224^{* *}$ & 0,6704 & 60,55 & 1,4426 & 0,4667 & - \\
\hline Comum & IV & $4247,982^{* *}$ & 0,9589 & 11,94 & 2,3800 & 0,1387 & - \\
\hline
\end{tabular}

$\mathrm{F}=\mathrm{F}$ calculado; $\mathrm{R}^{2}=$ coeficiente de determinação; $\mathrm{S}_{\mathrm{yx}} \%=$ erro padrão da estimativa em percentagem e Índice de furnival para os modelos logarítmicos; ${ }^{\star \star}=$ significante ao nível de $1 \%$ de probabilidade.

Portanto estas famílias contribuíram nas duas regiões com mais de $43 \%$ do total de indivíduos amostrados na classe de DAP $\geq 20$. Este resultado também foi encontrado por Richards (1957), que constatou, em floresta tropical, o predomínio de poucas familias sob o maior número de individuos como expressão de dominância da família na área.

As familias Violaceae, Sterculiaceae, Araceae, Boraginaceae, Nyctaginaceae, Araliaceae, Icacinaceae, Piperaceae, Sapindaceae, Musaceae, Gentiniaceae, Simarubaceae, Combretaceae, Verbenaceae, Myristicaceae, Bixaceae, Elaeocarpaceae, Humiriaceae apresentam-se com uma única espécie cada, o que representa cerca de $44 \%$ do total de famílias existentes nas regiões e cerca de $8,93 \%$ em Carajás e 2,40\% em Marabá das espécies existentes no nível I de amostragem. Destas, as famílias Piperaceae, Icacinaceae, Gentiniaceae, Verbenaceae e Humiriaceae são exclusivas da região de Carajás.

Pelo indice de agregação de McGuiness (IGA), nas duas regiões estudadas, as espécies com IVIAR ${ }^{*}>2$ ocorrem agregadas, sendo que na região de Carajás apenas $30 \%$ das espécies apresentam-se agregadas e outros $70 \%$ com tendência a agregação. Na região de Marabá mais de $80 \%$ das espécies encontram-se agregadas.

* IVIAR - Índice de valor de importância ampliado relativo 
Tabela 5. Relação das espécies encontradas nas regiōes estudadas.

\begin{tabular}{|c|c|c|}
\hline NOME VULGAR & NOME CIENTIFICO & FAMÍLIA \\
\hline Abiurana & Micropolis williamii Aubr. et Pellegr. & Sapotaceae \\
\hline Abiurana abiu & Pouteria guyanensis Aubl. & Sapotaceae \\
\hline Abiurana bacuri & Chrysophyllum guyanensis (Eyma)Baemi & Sapotaceae \\
\hline Açaí & Euterpe oleracea Mart. & Arecaceae \\
\hline Acapu & Vouacapoua pallidior Ducke & Caesalpiniaceae \\
\hline Acariquara roxa (") & Minquartia guianensis Aubl. & Olacaceae \\
\hline Achicha & Sterculia sp. & Sterculiaceae \\
\hline Alchornia & Indeterminada 1 & Euphorbiaceae \\
\hline Algodão bravo & Bombacopsis nervosa (Uitt) A. Robyns & Bombacaceae \\
\hline Anani & Symphonia globulifera L. & Clusiaceae \\
\hline Angelim da mata & Hymenolobium excelsum Ducke & Fabaceae \\
\hline Angico & Schizolobium sp. & Mimosaceae \\
\hline Araça da mata & Psidium araca Raddi & Myrtaceae \\
\hline Babaçu & Orbignya speciosa (Mart.) Barb. Rodr. & Arecaceae \\
\hline Bacaba & Oenocarpus bacaba Mart. & Arecaceae \\
\hline Bacuri & Platonia insignis Mart. & Clusiaceae \\
\hline Balsamo & Myroxyion sp. & Fabaceae \\
\hline Barauna & Indeterminada 2 & \\
\hline Branquinha & Rinorea racemosa (Mart. et Zucc.) O. Ktze & Violaceae \\
\hline Breu & Protium sp. & Burseraceae \\
\hline Breu vermelho & Protium sp. & Burseraceae \\
\hline Burra leiteira & Sapium marmieri Hub. & Euphorbiaceae \\
\hline Buxuxu & Miconia sp. & Melastomataceae \\
\hline Cacaui & Theobroma sylvestris Aubl. et Mart. & Sterculiaceae \\
\hline Caja & Spondias lutea $\mathrm{L}$. & Anacardiaceae \\
\hline Capa bode & Indeterminada 3 & \\
\hline Caraipé & Licania sp. & Chrysobalanaceae \\
\hline Caraiperana & Licania sp. & Chrysobalanaceae \\
\hline Carapanaúba & Aspidosperma carapanauba Pichon. & Apocynaceae \\
\hline Caroba & Jacaranda copaia D. Don. & Bignoniaceae \\
\hline Casca preciosa & Aniba canellila (H.B.K.) Mez. & Lauraceae \\
\hline Castanha de porco & Glycidendron amazonicum Ducke & Euphorbiaceae \\
\hline Castanha do pará & Bertholetia excelsa Humb. \& Bonpl. & Lecythidaceae \\
\hline Castanha fedorenta & Lipiosma sp. & Olacaceae \\
\hline Castanha jarana (*) & Lecythis sp. & Lecythidaceae \\
\hline Caucho & Castiloa ulei Warburg & Moraceae \\
\hline Cedro vermelho & Cedrela sp. & Meliaceae \\
\hline Chiclete bravo & Micropholis guyanensis Pierre & Sapotaceae \\
\hline Cipó & Indeterminada 4 & Araceae \\
\hline Coração de negro & Swartzia corrugata Benth. & Caesalpiniaceae \\
\hline Cumaru ferro & Dipteryx magnifica Ducke & Fabaceae \\
\hline Desconhecida & Indeterminada 5 & \\
\hline Embaúba & Cecropia sp. & Cecropiaceae \\
\hline Embaubarana & Pourouma longipendula Ducke & Cecropiaceae \\
\hline Envira & Guatteria sp. & Annonaceae \\
\hline Envira amarela & Guatteria sp. & Annonaceae \\
\hline Envira preta & Guatteria sp. & Annonaceae \\
\hline Falsa rainha & Clarisia sp. & Moraceae \\
\hline Faveira $\left(^{*}\right)$ & Hymenolobium sp. & Mimosaceae \\
\hline Freijó & Cordia goeldiana Hub. & Boraginaceae \\
\hline Gameleira & Ficus sp. & Moraceae \\
\hline Jenipapo & Genipa americana L. & Rubiaceae \\
\hline Gitó & Guarea sp. & Meliaceae \\
\hline
\end{tabular}


Tabela 5. Continuação.

\begin{tabular}{|c|c|c|}
\hline NOME VULGAR & NOME CIENTIFICO & FAMÍLIA \\
\hline Gogó de guariba & Rauwolfia paraensis Ducke & Apocynaceae \\
\hline Goiaba de anta & Bellucia grossularioides (L.) Triana & Melastomataceae \\
\hline Goiabinha & Myrciaria floribunda (Willd.) Berg. & Myrtaceae \\
\hline Guariúba $\left(^{*}\right)$ & Clarisia racemosa $R$. et $P$ & Moraceae \\
\hline Inajá & Maximiliana maripa (Aublet.) Driode & Arecaceae \\
\hline Ingá & Inga heterophylla Willd. & Mimosaceae \\
\hline Inharé & Helicostilys podogyne Ducke & Moraceae \\
\hline Itaúba $\left(^{*}\right)$ & Mezilaurus itauba (Meissn.) Taubert. ex Mez. & Lauraceae \\
\hline Jarai & Glycoxylon pedicellatum (Ducke) Ducke & Sapotaceae \\
\hline Jatoba & Hymenaea courbaril L. & Caesalpiniaceae \\
\hline Joäo mole & Neea sp. & Nyctaginaceae \\
\hline Jurema & Pityrocarpa ptervolada (Benth.) & BrenanMimosaceae \\
\hline Jutaicica & Dialium guianensis (Aubl.) Sandwith. & Caesalpiniaceae \\
\hline Lacre & Vismia guianensis (Aubl.) Choisy. & Clusiaceae \\
\hline Louro $\left({ }^{*}\right)$ & Ocotea sp. & Lauraceae \\
\hline Louro preto & Dicypelium manausense W. Rodr. & Lauraceae \\
\hline Macacauba $\left({ }^{\star}\right)$ & Platymiscium duckei Huber & Fabaceae \\
\hline Macucu & Licania sp. & Chrysobalanaceae \\
\hline Macucu fofo & Licania micrantha Miq. & Chrysobalanaceae \\
\hline Mamãorana & Catostema albuquerquei Paula & Bombacaceae \\
\hline Mamãozinho & Mouriri trunciflora Ducke & Memecylaceae \\
\hline Marupá (*) & Moronobea coccinea Aubl. & Clusiaceae \\
\hline Matamata & Eschweilera sp. & Lecythidaceae \\
\hline Mogno(*) & Swietenia macrophylla King. & Meliaceae \\
\hline Morototo & Didymopanax morototoni (Aubl.) Decne \& Planch & Araliaceae \\
\hline Muiracatiara $\left({ }^{*}\right)$ & Astronium $\mathrm{sp}$ & Anacardiaceae \\
\hline Muirachimbe & Emmotum sp. & Icacinaceae \\
\hline Muirajibóia preta & Bocoa sp. & Caesalpiniaceae \\
\hline Muiratinga $\left({ }^{*}\right)$ & Naucleopsis sp. & Moraceae \\
\hline Mururé & Bombacopsis sp. & Bombacaceae \\
\hline Mutamba & Brosimum sp. & Moraceae \\
\hline Pajurazinho & Licania sp. & Chrysobalanaceae \\
\hline Parica & Schizolobium sp. & Caesalpiniaceae \\
\hline Pau chichua & Maytenus sp. & Celastraceae \\
\hline Pau d'arco $\left({ }^{*}\right)$ & Tabebuia sp. & Bignoniaceae \\
\hline Pau mulato & Peltogyne sp. & Caesalpiniaceae \\
\hline Pau paratudo & Simaruba sp. & Simarubaceae \\
\hline Pau pombo & Tapirera sp. & Anacardiaceae \\
\hline Pau pretinho & Cenostigma sp. & Caesalpiniaceae \\
\hline Pau rainha( $\left(^{*}\right)$ & Brosimum rubescens Taub. & Moraceae \\
\hline Pau tanino & Maquira sp. & Moraceae \\
\hline Paxiuba & Socratea sp. & Arecaceae \\
\hline Piabinha & Casearia sp. & Flacourtiaceae \\
\hline Pimenta longa & Piper sp. & Piperaceae \\
\hline Pintadinho & Licania sp. & Chrysobalanaceae \\
\hline Piquia marfim & Aspidosperma album (Vahl.) R. Bem. & Apocynaceae \\
\hline Piquia verdadeiro & Caryocar villosum (Aubl.) Pers. & Caryocaraceae \\
\hline Piranheira & Piranhea sp. & Euphorbiaceae \\
\hline Pirarara & Indeterminada 6 & \\
\hline Periquiteira & Laetia sp. & Flacourtiaceae \\
\hline Pitomba da mata & Talisia sp. & Sapindaceae \\
\hline Pracuuba & Lecointea sp. & Caesalpiniaceae \\
\hline Purui & Clusia sp. & Clusiaceae \\
\hline
\end{tabular}


Tabela 5. Continuação.

\begin{tabular}{|c|c|c|}
\hline NOME VULGAR & NOME CIENTIFICO & FAMÍLIA \\
\hline Sapateiro & Tovomita sp. & Clusiaceae \\
\hline Seringarana & Mabea sp. & Euphorbiaceae \\
\hline Sororoca & Ravenala sp. & Musaceae \\
\hline Sorva brava & Couma sp. & Apocynaceae \\
\hline Sucupira $\left(^{*}\right)$ & Bowdichia sp. & Fabaceae \\
\hline Sucupira preta & Diplotropis sp. & Fabaceae \\
\hline Sumaúma & Ceiba pentandra Gaertn. & Bombacaceae \\
\hline Tabaco bravo & Chelonanthus sp. & Gentianaceae \\
\hline Taboquinha & Palicourea sp. & Rubiaceae \\
\hline Tachi & Sclerolobium sp. & Caesalpiniaceae \\
\hline Tamanqueira & Simaruba sp. & Simarubaceae \\
\hline Tanimbuca & Buchenavia sp. & Combretaceae \\
\hline Tapereba & Spondias lutea L. & Anacardiaceae \\
\hline Tarumă & Vitex sp. & Verbenaceae \\
\hline Tatajuba & Bagassa sp. & Moraceae \\
\hline Tauari & Cariniana sp. & Lecythidaceae \\
\hline Tento preto & Osmosia sp. & Fabaceae \\
\hline Tucumä & Astrocaryum sp. & Arecaceae \\
\hline Ucuuba preta(*) & Virola sp. & Myristicaceae \\
\hline Urucum bravo & Cochlospermum sp. & Bixaceae \\
\hline Urucurana & & Elaeocarpaceae \\
\hline Uxi & Endopleura sp. & Humiriaceae \\
\hline
\end{tabular}

$\left(^{\star}\right)$ - espécies listadas (com vator econômico no mercado)

\section{Estrutura Horizontal}

Através da análise dos resultados da Tabela 6 constata-se que 20 espécies contribuíram com $(63,89 \%)$ na micro-região de Carajás e $(61,43 \%)$ em Marabá, da soma total do IVI; estes dados não diferem muito do IVIA que ficou em torno de $(66,25 \%)$ para Carajás e $(62,46 \%)$ para Marabá. Pode-se verificar também que a hierarquia de ordenação das espécies não varia muito em termos de IVI e VC* . Dentre as espécies com maiores valores de IVI destacamos: Breu Vermelho, Pau Pretinho, Ingá, Castanha do Pará, Babaçu e Jurema em Carajás; e Breu Vermelho, Gitó, Ingá, Castanha do Pará, Achicha, Sapateiro e Burra Leiteira na região de Marabá.
Ainda podemos inferir desses resultados que $89(72,95 \%)$ das espécies na região de Marabá e 96 $(73,85 \%)$ em Carajás apresentaram IVI menor que $2 \%$. Este resultado foi similar aos obtidos por Martins (1979) que menciona ser caracteristica de florestas tropicais a presença de um número elevado de espécies com IVI baixo.

\section{Estimativa dos Parâmetros da Estrutura Vertical}

As estimativas dos parâmetros da estrutura vertical englobam as estimativas de posição sociológica e regeneração natural relativa (Tab. 6).

A estimativa da posição sociológica foi efetuada tendo por base o valor fitossociológico da espécie.

- VC - Valor de cobertura 
Tabela 6. Estimativas dos parâmetros fitossociológicos das espécies de IVIAR>2.

\begin{tabular}{llccccccccc}
\hline REGIÃO & ESPÉCIE & VC & VCR & IVI & IVIR & PS & PSR & RNR & IVIA & IVIAR \\
\hline Carajás & Breu vermelho & 35,352 & 17,677 & 39,939 & 13,939 & 21,163 & 19,347 & 6,740 & 66,026 & 13,206 \\
& Pau pretinho & 24,018 & 12,010 & 28,186 & 9,396 & 15,191 & 13,887 & 3,281 & 45,354 & 9,071 \\
& Ingá & 14,235 & 7,118 & 19,099 & 6,367 & 10,467 & 9,569 & 8,433 & 37,101 & 7,421 \\
& Babaçu & 10,137 & 5,069 & 12,637 & 4,213 & 6,044 & 5,525 & - & 18,162 & 3,633 \\
& João mole & 4,447 & 2,224 & 6,811 & 2,270 & 3,824 & 3,496 & 6,104 & 16,411 & 3,282 \\
& Castanha do Pará & 14,789 & 7,395 & 16,316 & 5,439 & 0,091 & 0,083 & - & 16,399 & 3,280 \\
& Gitó & 3,697 & 1,849 & 6,756 & 2,252 & 2,727 & 2,493 & 4,277 & 13,526 & 2,705 \\
& Jurema & 5,928 & 2,964 & 9,265 & 3,088 & 3,158 & 2,887 & 0,826 & 12,978 & 2,596 \\
& Abiurana & 5,155 & 2,578 & 8,073 & 2,691 & 2,261 & 2,067 & 1,592 & 11,732 & 2,347 \\
& Ucuuba preta & 3,964 & 1,982 & 6,605 & 2,202 & 2,473 & 2,261 & 1,492 & 10,358 & 2,072 \\
Marabána & Breu vermelho & 41,573 & 20,786 & 45,444 & 15,147 & 18,756 & 19,960 & 5,586 & 70,990 & 14,197 \\
& Ingá & 12,444 & 6,222 & 16,703 & 5,567 & 8,351 & 8,887 & 5,808 & 31,398 & 6,279 \\
& Gitó & 12,863 & 6,431 & 16,930 & 5,643 & 8,571 & 9,121 & 5,132 & 31,183 & 6,236 \\
& Castanha do Parána & 14,492 & 7,246 & 16,813 & 5,604 & 0,328 & 0,349 & 0,741 & 17,903 & 3,580 \\
& Seringarana & 5,581 & 2,790 & 8,496 & 2,828 & 0,096 & 0,102 & 8,065 & 16,653 & 3,330 \\
& Matamata & 4,158 & 2,079 & 7,254 & 2,418 & 2,439 & 2,596 & 2,721 & 12,571 & 2,514 \\
& Achicha & 5,933 & 2,966 & 9,029 & 3,009 & 3,059 & 3,255 & - & 12,284 & 2,457 \\
& Breu & 4,189 & 2,092 & 6,701 & 2,233 & 2,370 & 2,522 & 2,693 & 11,916 & 2,383 \\
& Envira & 3,378 & 1,689 & 6,474 & 2,158 & 2,425 & 2,581 & 2,589 & 11,644 & 2,329 \\
& Sororoca & - & - & - & - & 4,630 & 4,927 & 5,692 & 10,619 & 2,124 \\
& Burra leiteira & 5,666 & 2,833 & 7,987 & 2,662 & 2,028 & 2,158 & - & 10,145 & 2,029 \\
\hline
\end{tabular}

LEGENDA:VC = Valor de cobertura; VCR = Valor de cobertura relativo; IVI = Indice de valor de importância; $\mid V I R$ $=$ IVI relativo; $P S=$ Posiçăo sociológica; $P S R=P S$ relativa; IVIA = Indice de valor de importância ampliado; IVIAR $=I V I A$ relativo.

Dentre as espécies de maior posição sociológica relativa assinala-se: Breu Vermelho (19.35\%); Pau Pretinho $(13,89 \%)$ e Ingá $(9.57 \%)$ em Carajás que representam mais de $42 \%$ do total de posição sociológica relativa da região. Na região de Marabá, as principais foram: Breu Vermelho (19,93\%), Gitó (9,11\%), Ingá $(8,88 \%)$ e Achicha $(3,25 \%)$ as quais representam $41 \%$ do total de PSR.

A estimativa da regeneração natural relativa foi obtida pela média aritmética dos parâmetros densidade, freqüência e classe de tamanho relativa do primeiro nível abordado. Em Carajás, as espécies: Castanha do Pará, Babaçu, Inajá, Cumaru Ferro e Faveira e em Marabá, as espécies:
Achicha, Burra Leiteira, Maçaranduba e Pau pombo apesar de encontrarem-se entre as de maiores valores de IVI, não possuem estoque de regeneração natural.

Verifica-se na Tabela 7 que as espécies Castanha do Pará e Babaçu estão ameaçadas de desaparecer da micro-região de Carajás, pois não foram encontrados individuos no intervalo de $5 \mathrm{~cm}$ a $20 \mathrm{~cm}$ de diâmetro à altura do peito.

$\mathrm{Na}$ área de Marabá, as espécies Achicha e Burra Leiteira também não possuem estoque de regeneração natural. Apenas duas espécies em Carajás, Breu Vermelho e Gitó e, Breu Vermelho, em Marabá, encontram-se representadas em todas as classes de tamanho e posição sociológica (CT e PS). 
Tabela 7. Estimativa do número de indivíduos por hectare para IVIAR $>2$.

\begin{tabular}{|c|c|c|c|c|c|c|c|c|}
\hline REGIÃO & ESPÉCIE & $\mathbf{N}$ & $\mathrm{CT}_{1}$ & $\mathrm{CT}_{2}$ & $\mathrm{CT}_{3}$ & PS $_{1}$ & $\mathrm{PS}_{2}$ & $\mathrm{PS}_{3}$ \\
\hline \multirow[t]{9}{*}{ Carajás } & Breu vermelho & 29,14 & 1,71 & 1,00 & 1,00 & 23,14 & 2,21 & 0,07 \\
\hline & Pau pretinho & 19,43 & 0,79 & 0,57 & 0,21 & 16,64 & 1,21 & - \\
\hline & Ingá & 16,43 & 2,57 & 1,43 & 0,50 & 11,50 & 0,43 & - \\
\hline & Babaçu & 6,86 & - & - & - & 6,64 & 0,21 & 2 \\
\hline & Joăo mole & 7,79 & 1,86 & 1,00 & 0,71 & 4,21 & - & $\cdot$ \\
\hline & Castanha do Pará & 1,21 & - & - & $\cdot$ & 0,07 & 0,07 & 1,07 \\
\hline & Gitó & 5,14 & 1,21 & 0,64 & 0,21 & 3,00 & 0,07 & 0,07 \\
\hline & Jurema & 4,43 & 0,22 & 0,14 & , & 3,43 & 0,64 & $\cdot$ \\
\hline & Abiurana & 3,86 & 0,50 & - & 0,07 & 2,43 & 0,79 & - \\
\hline \multirow[t]{11}{*}{ Marabá } & Breu vermelho & 29,77 & 1,59 & 1,02 & 0,34 & 21,36 & 5,11 & 0,23 \\
\hline & ingá & 13,07 & 1,14 & 1,25 & 0,45 & 9,77 & 0,45 & - \\
\hline & Gitó & 13,30 & 1,25 & 0,45 & 0,91 & 10,00 & 0,68 & - \\
\hline & Castanha do Pará & 1,93 & $\cdot$ & 0,23 & 0,11 & 0,34 & $\cdot$ & 1,25 \\
\hline & Seringarana & 13,41 & 5,00 & 2,27 & 0,68 & 5,45 & - & - \\
\hline & Matamata & 4,20 & 0,68 & 0.11 & 0,34 & 2,84 & 0,23 & - \\
\hline & Achicha & 4,09 & - & - & - & 3,52 & 0,57 & - \\
\hline & Breu & 4,32 & 0,80 & 0,23 & 0,11 & 2,73 & 0,45 & - \\
\hline & Envira & 4,09 & 0,57 & 0,57 & - & 2,84 & 0.11 & - \\
\hline & Sororoca & 0,23 & $\cdot$ & 0,11 & 0,11 & $\cdot$ & $\cdot$ & - \\
\hline & Burra leiteira & 3,18 & - & $\cdot$ & - & 2,27 & 0,80 & 0,11 \\
\hline
\end{tabular}

LEGENDA: $\mathrm{N}=$ Número total de indivíduos/ha; $\mathrm{CT}_{1}, \mathrm{CT}_{2}$ e $\mathrm{CT}_{3}=$ Número de individuos/ha nas classes de tamanho 1,2 e $3 ; \mathrm{PS}_{1}, \mathrm{PS}_{2}$ e $\mathrm{PS}_{3}=$ Número de individuos nas posiçốes sociológicas 1,2 e 3.

\section{Índices de Diversidade e Similaridade}

A estimativa de diversidade florística das duas micro-regiões, considerando o Índice de ShannonWeaver, ficou em torno de 3,66 para a área de Carajás e 3,71 para a região de Marabá, resultados semelhantes também foram encontrados por Barros (1980) em estudos conduzidos em floresta tropical úmida na Amazônia brasileira, encontrando uma diversidade estimada em 4,8 pelo "Índice de Shanon-Weaver". Na Amazônia venezuelana UHL et al., (1981) encontraram valores que variam de 4,8 a 5,4 . Ressalta-se, que, quanto maior esse índice, maior será a diversidade florística da área em questão.
$\mathrm{Na}$ análise comparativa das duas micro-regiões utilizou-se o Índice de Sorensen, que, apesar de possuir a desvantagem de ser mais qualitativo, pode dar uma idéia a respeito da similaridade das regiões. Neste trabalho esta estimativa ficou em 0,73 , ou seja, existe $73 \%$ de possibilidade das áreas serem similares.

\section{Índice de Agregação}

Pelo índice de agregação de McGuiness (IGA), nas duas regiões estudadas, as espécies com IVIAR $>2$ ocorrem agregadas, sendo que na região de Carajás apenas 30\% das espécies apresentam-se agregadas e outros $70 \%$ com tendência a agregação. Na região de Marabá mais de $80 \%$ das espécies encontram-se agregadas. 


\section{Distribuição Diamétrica}

A distribuição diamétrica das regiões estudadas obedece o padrão das florestas ineqüiâneas, isto é, padrão exponencial em " $\mathrm{J}$ " invertido (Meyer $\mathrm{et}$. al., 1961). Na Tabela 8 encontram-se os modelos testados e os parâmetros obtidos através de regressão não linear para as duas micro-regiões estudadas. Nas Figuras 2 e 3 observa-se a distribuição diamétrica encontrada e os respectivos valores estimados pelos parâmetros da regressão.

\section{CONCLUSÕES}

Apesar dos resultados obtidos serem exclusivos para as regiões de Carajás e Marabá, os mesmos, devem servir como base para comparações com outras regiões semelhantes da Amazônia;

Das espécies que apresentam os maiores valores de IVIA, as primeiras posições sempre estão ocupadas por espécies que atualmente não possuem importância econômica, caracterizando dessa forma um baixo valor madeireiro para a floresta das regiões;

Tabela 8. Características dos modelos de distribuição diamétrica analisados.

\begin{tabular}{|c|c|c|c|c|c|c|c|}
\hline REGIÃO & MODELOS & $\mathbf{F}$ & $\mathbf{R}^{2}$ & $\mathrm{~S}_{\mathrm{yX}} \%$ & $\beta_{0}$ & $\beta_{1}$ & $\beta_{2}$ \\
\hline \multirow[t]{2}{*}{ Carajás } & $F_{1}=e^{(\beta 0+\beta(\beta 1 d)} \cdot A \tilde{A} i$ & $10,149^{\star \star}$ & 0,7531 & 14,33 & 4,2959 & 1,57 & 0,36 \\
\hline & $F_{i}=c \cdot(x-a)^{\alpha} \cdot(b-x)^{\beta}$ & $1304,1^{\star \star}$ & 0,9968 & & 9,56330 & 1,00 & 0,014 \\
\hline \multirow[t]{2}{*}{ Marabá } & 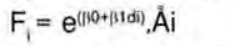 & $17,625^{\star *}$ & 0,8981 & 8,81 & 3,7968 & 2,73 & 1,93 \\
\hline & $F_{1}=c \cdot(x-a)^{\alpha} \cdot(b-x)^{\beta}$ & $962,8^{\star \star}$ & 0,9953 & & & - & 0,34 \\
\hline
\end{tabular}

LEGENDA: $\mathrm{F}=\mathrm{F}$ calculado; $\mathrm{R}^{2}=$ Coeficiente de determinação; $\mathrm{S}_{\mathrm{yx}} \%=$ Erro padrão da estimativa em percentagem; ${ }^{* \star}=$ Significante ao nível de $1 \%$ de probabilidade.

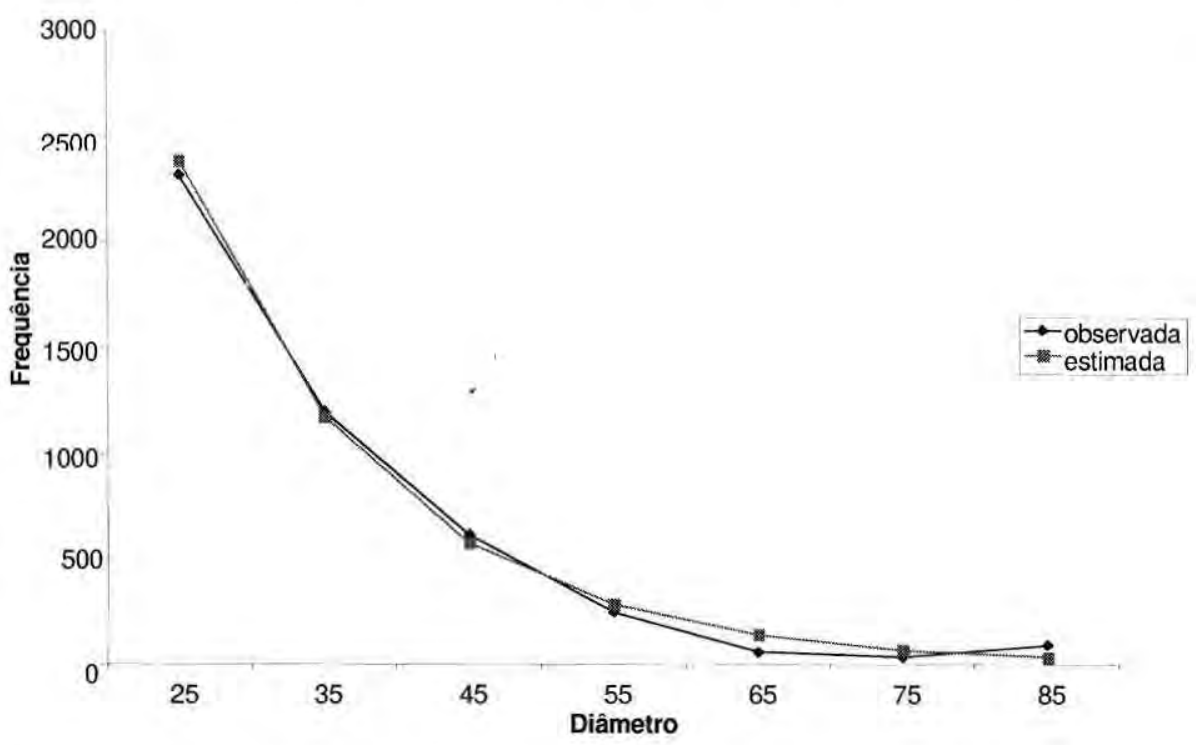

Figura 2. Distribuição diamétrica para a região de Carajảs - PA. 


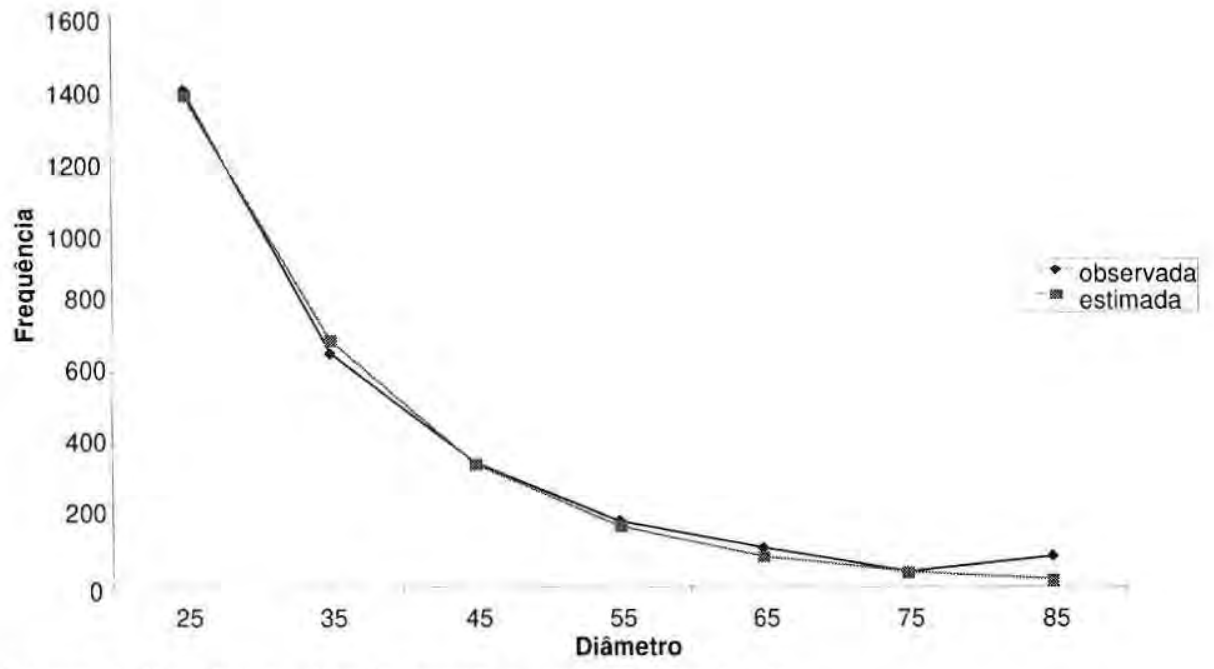

Figura 3. Distribuição diamétrica para a região de Marabá-PA.

A estrutura e fisionomia ora existente nas regiões estudadas são conseqüências de fatores de perturbação ambiental mais especificamente ao intenso abate seletivo;

Nas regiões estudadas verifica-se que a distribuição diamétrica apresenta-se com a forma de " $\mathrm{J}$ " invertido, característica peculiar de floresta ineqüânea heterogênea;

A equação que melhor ajustou os dados deste trabalho foi a função de densidade I (Meyer) dada pelas seguintes equações: $F_{i}=e^{(0,56330614-0,07139847 \mathrm{~d})}$ para Carajás e $\mathrm{F}_{i}=\mathrm{e}^{(8,96125691 \cdot 0,06937877 \mathrm{~d})}$ para Marabá;

A equação hipsométrica recomendada por este trabalho é a seguinte: $h=\left[\frac{d}{2,3800+0,1387 d}\right]^{2}$

Os resultados da composição florística e estrutura fitossociológica indicaram que as duas regiões apresentam elevada diversidade floristica, apresentando um total de 130 espécies em Carajás e 122 espécies em Marabá e com Índice de
Shanon-Weaver de 3,66 e 3,71, respectivamente;

A área basal média estimada para Carajás é de $15,41 \mathrm{~m}^{2} /$ ha e a abundância média de 131,92 indivíduos/ha. Em Marabá a área basal média é de $17,35 \mathrm{~m}^{2} /$ ha e a abundância média de 127,85 indivíduos/ha;

$\mathrm{Na}$ região de Carajás estima-se que cerca de $18 \%$ das espécies são utilizadas para exportação e outros $18 \%$ são para utilização nacional. Em Marabá esta estimativa fica em torno de $19 \%$ e $17 \%$ respectivamente para exportação e nacional;

Nas regiões estudadas verifica-se que a maioria das espécies apresentam padrões de distribuição agregado ou tendendo ao agregamento o que vem sugerir que encontram-se em estágio de sucessão secundária;

A espécie mais importante na estrutura florestal, para as regiões de Carajás e Marabá, é o Breu Vermelho. Além disto, os resultados permitem formular algumas recomendações: 
Aproveitamento econômico da floresta, com base nos princípios ecológicos, isto é, sem provocar grandes alterações na sua estrutura atual;

Identificar as causas da regeneração natural deficiente das espécies valiosas, com o objetivo de favorecê-las, antes que sejam substituídas por outras espécies; e

Efetuar o acompanhamento do crescimento das árvores através de parcelas permanentes.

\section{AGRADECIMENTOS}

Os autores agradecem ao Ministério de Ciência e Tecnologia, Instituto Nacional de Pesquisas da Amazônia pelo financiamento deste trabalho. À Maria Naélia Silva Ribeiro e José Ferreira Ramos da Coordenadoria de Pesquisas em Botânica pela ajuda na parte florística, além de Paulo Ary da Silva Santana da Coordenadoria de Pesquisas em Silvicultura Tropical, pela edição do texto.

\section{Bibliografia citada}

Atlas do Conselho Interministerial do Programa Grande Carajás. 1981. Programa Grande Carajás; aspectos fisicos, demográficos e fundiários, Rio de Janeiro.

Ab Saber, A.N. 1986. Geomorfologia da região, In: Carajás: desafio politico, ecologia e desenvolvimento, São. Paulo: Brasiliense; Brasilia; CNPq, p. 88-124.

Barnard, R. C. 1950. Linear regeneration sampling. The Malay, For, Kuala Lumpur, 13(3): 129-142.

Barros, P. L. C. 1980. Estudo das distribuições diamétricas da Floresta do Planalto Tapajós-Pará. Dissertação de Mestrado. UFPr. Curitiba, Paraná.

Carvalho, J. O. P. de; 1982. Análise estrutural da Regeneração Natural em Floresta Tropical densa na região do Tapajós no
Estado do Pará. Dissertação de Mestrado. UFPr, Curitiba.

Calegário, N. 1993. Parâmetros floristicos e fitossociológicos da Regeneração Natural de espécies arbóreas nativas no sub bosque de Povoamentos de Eucapyptus, no Municipio de (Belo Oriente)/MG. Viçosa, UFV, 114 p,il.

Cunha, O. R. da; Nascimento, F. P.; Avila-Pires, T. C. S. 1985. Os répteis da área de Carajás, Pará, Brasil, Publ. Avuls. Mus. Par. Emilio Goeldi, Belém, 40: 9-92

Curtis, J. T.; McIntosh, R. P. 1951. An upland forest continuum in the prairies forest border region of Wisconsin. Ecology, 32: 478-496

Dansereau, P. 1961. Essais de représentation cartographique des eléments structuraux de la végétation. In: Méthodes de la cartographie de la végétation. Toulouse, Centre Nacional de la Recherche Scientifique. p. 233-255.

Finol, U. H. 1971. Nuevos parâmetros a considerar-se em el análisis estrutural de las selvas virgenes tropicales, $R$. For.Venez., 14(21): 29-42.

Flores, E. J. M. 1993. Potencial produtivo e alternativo de manejo sustentável de um fragmento de Mata Atlântica Secundária, Municipio de Viçosa. Minas Gerais. UFV, 165 p. il.

Graybill, F. A. 1976. Theory and application of the linear model, USA, Duxbury Press, 704p.

Higuchi, N.; Jardim, F. C. S.; Santos, J. dos; Barbosa, A. P.; Wood, T. W. W. 1985b. Bacia 3: Inventário Florestal Comercial. Acta Amazonica, 15(3-4): 327-69.

Higuchi, N.; Santos, J. dos; Jardim, F. C. S. 1982. Tamanho de parcela amostral para Inventários Florestais. Acta Amazonica, 12(1): 91-103

Higuchi, N.; Salati, E.; Filho, L. G. M.; Santos, J. M. dos; 1992. Estimativa da Fitomassa (acima do nivel do solo) na região sul do Pará. Relatório CPST/INPA, 53p.

Horn, H. S. 1966. Measurement of "overlap" in comparative ecological studies, The American Naturalist. 100(914):424. 
Hueck, K. 1972. As florestas da América do Sul. São Paulo, Poligono, Brasilia, Ed, Univ. Brasilia, p. 26.

Husch, B.; Miller, C. J.; Beers, T. W. 1972. Forest mensuration. The Ronald press, $410 \mathrm{p}$.

Jardim, F. C. S.; Hosokawa, R.T. 1986/1987. Estrutura da floresta equatorial úmida da estação experimental de silvicultura tropical do INPA. Acta Amazonica, 16/ 17(único): 411-508.

Lamprecht, H. 1964. Ensayo sobre la estrutura floristica de la parte sur-oriental del Bosque Universitário "El caimital", Estato Barinas. Ver: For: Venez., 7(10-11): 77-119.

Longhi, S. J. 1980. A estrutura de uma floresta natural de Araucaria angustifolia (Bert,) $O$, Ktze, sul do Brasil. Curitiba, Dissertação de Mestrado, UFPr, 198 p.

Martins, F. R. 1979. O método de quadrantes e a fitossociologia de uma floresta residual no interior do estado de São Paulo: Parque Estadual de Vassunga. São Paulo (SP), USP, 239 p.

Meyer, H. A.; Recknagel, A. B.; Stevenson, D. D.; Bartoo, R. A. 1961. Forest Management. New York, Ronald Press, 282 p.

Montoya Maquin, J. M. 1966. El acuerdo de yangambi (1956) como base para una nomenclatura de tipos de vegetacion en el tropico americano. Turrialba, 17(2): 197-207.

Péllico Neto, S. 1982. Inventário Florestal. Centro Acadêmico de Engenharia Florestal. UFPr. Apostila. 110p.

Pereira, M. M. 1991. Caracterização do clima da micro-região de Marabá e identificação das áreas adaptadas ao cultivo de arroz, milho, feijão caupi e castanha sobre o ponto de vista climatológico. UFP. 30p.
Poole, R. W. 1974. A introduction to quantitative ecology. New York, McGraw-Hill, 532p.

Porto, M. L.; Longhi, H. M.; Citadini, V.; Ramos, R. F.; Mariath, J. E. A. 1976, Levantamento fitossociológico em área de "mata de baixio" na Estaçào Experimental de Silvicultura Tropical, INPA. Acta Amazonica, 6(3): 301-318.

Relatório da CVRD. 1980. Relatório de reavaliação da jazida de ferro, N4-E do Distrito Ferrifero da Serra dos Carajás. Belo Horizonte, Companhia Vale do Rio Doce, V, I.

Relatório do Museu Goeldi. 1988. A companhia Vale do Rio Doce. Belém (PA), V, I.

Richards, P. W. 1957. The tropical rain forests; an ecological study. Cambridge, University Press, 450p.

Salomão, R. de P.; Rosa, N. de A. 1989. Análise da vegetação de floresta pluvial tropical de terra firme, pelo método dos quadrantes, Serra Norte Carajás, PA.

Scolforo, J. R. S.; Chaves, A. L.; Melo, J. M. de; 1993. Defínição de tamanho de parcela para inventário florestal em floresta semidecidua montana. Anais VII Cong, Flor: Bras., 333-337.

Uhl, C.; Clark, H.; Murphy, P. G. 1981. Early plant sucession after cutting and burning in the upper Rio Negro region the Amazon Basin. $J$. Ecol., 69(2): 631-49.

Vieira, G. 1987. Análise estrutural da regeneração natural, após diferentes niveis de exploração em uma floresta tropical úmida. Dissertação de Mestrado. INPA/FUA, Manaus. 161p. 